\title{
Breaking Out of the Silo: Education across Disciplines in Restoration Ecology
}

A $s$ both Mrill Ingram and James Aronson point out in their editorials in this special issue, ecological ing drawn from a wide range of sources from very different disciplines. Sometimes these disciplines are closely related, such as plant and soil sciences; sometimes they are disparate, such as economics and hydrology_or least it would appear so. As ecologists, we know just how important it is to have a knowledge of these different components of restoration in order to even start to put things back together-Tony Bradshaw famously called ecological restoration the "acid test" of our knowledge of ecosystem structure and function. Ecological restoration is incomplete without the embedding of our activities in the society in which we live at a number of scales, from the local lot to international agreements-we are, after all, attempting to reconnect nature and culture.

So why are ecological restoration programs taught at all levels apparently so thin on the ground? We have several examples of outstanding programs in this present issue, so it isn't impossible - but what is preventing a wider uptake? I think, in part, this difficulty arises from the way in which research is funded, and academic careers assessed, in universities and colleges. Research portfolios are often the principal means by which appointments and promotions are made, and these are heavily dependant on publication in high-impact journals. The highest-impact journals generally have a single-discipline focus (outside of Science, Nature, and the likes of TREE) - this is why $E R$ and Restoration Ecology are such important journals for our community.
This takes us to the next issue: the difficulties of offering courses outside of narrow single-discipline approaches and the need for disparate disciplines within the corpus of a faculty (and beyond) to collaborate on delivering undergraduate and postgraduate programs. This works exceedingly well in some places and not at all in others. In the latter, it is incumbent upon educators to expand outside of their narrow disciplines in developing teaching and research programs - we have to go the extra mile if we are to be successful. There is a lot of evidence (see this present special issue) that this approach is successful and rewarding on a number of levels, and the fact is that a rapid expansion in the number of programs is already beginning.

The final piece in this puzzle is how do we attract students to these courses and make them viable? We all have a duty to enthuse those around us and beyond. The Society for Ecological Restoration International certainly has a role in this by raising public awareness of an optimistic agenda in the environmental sphere, where we can and should act to repair our damaged ecosystems-young people are not only keen to take this forward, but realize that it's their future that we are aiming to secure. Let's go and engage with them.

\section{Acknowledgment}

I thank Cara Nelson for thoughtful comments on this piecehowever, any sins of omission or commission are wholly mine!

\author{
Jim Harris \\ Chair \\ Society for Ecological Restoration International
}

